\title{
Sistema inteligente para procesamiento de imágenes en control de calidad basado en el modelo difuso singleton tipo 1
}

\author{
Pascual Noradino Montes Dorantes ${ }^{1}$, Juan Pablo Nieto González ${ }^{1}$, \\ Gerardo Maximiliano Méndez ${ }^{2}$, Rolando Praga Alejo ${ }^{1}$, Karla Lizette Guajardo Cosio ${ }^{1}$ \\ ${ }^{1}$ Corporación Mexicana de Investigación en Materiales S.A. de C.V., \\ Saltillo, Coahuila, México \\ ${ }^{2}$ Posgrado en Ingeniería Mecatrónica, Instituto Tecnológico de Nuevo León, \\ Guadalupe, Nuevo León, México \\ pascualresearch@gmail.com; juan.nieto@comimsa.com; \\ gmm_paper@yahoo.com.mx; rolandopraga@comimsa.com; Karla.guajardo@comimsa.com
}

\begin{abstract}
Resumen. En este artículo se presenta una metodología hibrida basada en un modelo difuso del tipo 1 en versión singleton usando un diseño factorial $2^{\mathrm{k}}$ que optimiza el modelo del sistema experto y sirve para realizar inspección en línea. El método de diseño factorial proporciona la base de datos necesaria para realizar la creación de la base de reglas para el modelo difuso y también genera la base de datos para entrenar el sistema experto. El método propuesto ha sido validado en el proceso de verificación de parámetros dimensionales por medio de imágenes comparándolo con los modelos ANFIS y RBFN los cuales muestran mayores márgenes de error en la aproximación de la función que representa el sistema comparada con el modelo propuesto. Los resultados obtenidos muestran que el modelo tiene un excelente desempeño en la predicción y el control de la calidad del proceso industrial estudiado cuando se compara con técnicas de sistemas expertos similares (ANFIS, RBFN).
\end{abstract}

Palabras clave: T1-SFLS, sistema difuso, diseño factorial, reducción de reglas, inspección de calidad, procesamiento de imágenes, visión artificial.

\section{$1 \quad$ Introducción}

La inspección de calidad hoy en día es aún un proceso que depende de las capacidades humanas. Junto al desarrollo de la ciencia y tecnología de la computación los procesos de inspección comenzaron a cambiar de la visión humana a la visión artificial. Un sistema de visión artificial se puede definir como el ensamble de una fuente de luz, un dispositivo que captura la imagen y un software que se dedique a procesarla [1]. Por otro lado, la minería de datos es muy importante para estos sistemas ya que, se requiere procesar una gran cantidad información proveniente de diferentes bases de datos, las cuales contienen múltiples campos de información que 
no es necesaria para el modelo. Para optimizar los sistemas es necesario elaborar una búsqueda de patrones para simplificar la complejidad [2], esta búsqueda reduce las demandas computacionales del sistema particularmente en la fase de adquisición del conocimiento.

Los sistemas de computación suave, sistemas inteligentes o sistemas expertos usan diferentes algoritmos para el manejo del conocimiento en el entrenamiento y la aproximación. Algunos de estos son: los algoritmos de Karnik-Mendel (KM), este algoritmo presenta complicaciones para generar una aproximación de la función que representa el proceso; debido a su naturaleza iterativa generalmente presenta retraso en el tiempo de repuesta [3]. Otros algoritmos comúnmente usados para el entrenamiento de los sistemas inteligentes son los siguientes: modelo de paso descendente (steepest descend) [4], mínimos cuadrados [4-5], modelo de gradiente descendente [5], agrupamiento (clustering) [5], estos presentaran naturaleza iterativa que provee el ajuste necesario para reducir el error. Adicionalmente, las herramientas que se han usado principalmente para unir el Diseño de experimentos (DOE) con la computación suave y control de calidad son: superficie de respuesta [6], Metodología de Taguchi, diseño factorial (DF) [7] y el diseño central compuesto (DCC) [8].

Los modelos de computación suave, particularmente la lógica difusa y las redes neuronales o modelos híbridos, brindan la posibilidad de evaluar dentro del sistema una gama de posibilidades prácticamente infinita; estos sistemas realizan su evaluación en un universo continuo (difuso) en lugar de un universo discreto (crisp) [9]. Debido a que no son restrictivos, los sistemas difusos se pueden simplificar por medio de una reducción en el tamaño de su base de reglas. No se requiere probar todos los estados del sistema ya que la dinámica de este tipo de sistemas expertos es capaz de aproximar comportamientos a partir de un patrón, en este caso en particular, esto se realiza por medio de una interpolación para obtener una conversión de los valores de la especificación a pixeles y obtener el valor de los limites en pixeles para poder generar las reglas difusas, esto sucede debido a que no en todas las ocasiones se dispone de los recursos necesarios para la realización de pruebas físicas (en los límites de control) ya que el producir estos ajustes a las maquinas para volver al control del proceso. Los resultados utilizados para los consecuentes se pueden simular a partir de datos históricos por medio de la interpolación mencionada. Es suficiente contar con las observaciones de los límites de la especificación: límite inferior de control (LIC) $\forall x_{i} \in X i=1,2, \ldots, n$, límite superior de control (LSC) $\forall x_{i} \in X i=1,2, \ldots, n$. en caso de no conocer todos los datos, estos se pueden aproximar por medio de alguna técnica matemática de aproximación a partir de los datos de la especificación que serán convertidos a pixeles. e.g. mínimos cuadrados.

Los sistemas de visión artificial usados industrialmente, particularmente los sistemas de adquisición, supervisión y control (SCADA) necesitan evolucionar adaptarse para ser más confiables. Además se debe lidiar con múltiples factores que afectan su desempeño. Estos factores generan variaciones en la magnitud de los datos de las entradas [10]. Esta incertidumbre puede originarse en diferentes fuentes como las características de las materias primas, producción, maquinaria, programación de la producción, los sistemas de adquisición de datos (sensores) y del ambiente principalmente [11]. Los sistemas la adquisición de los datos constituyen una parte 
fundamental para la evaluación, el monitoreo y el control de los procesos de manufactura, el cual es afectado continuamente por la incertidumbre [12]. Por otro lado el modelo de Taguchi toma en cuenta la incertidumbre y la clasifica en tres formas: interna, externa y de producción [13]. En base a estas dos perspectivas se puede definir la siguiente clasificación:

- Incertidumbre interna, este procede de las materias primas y la principal causa de esta clase de incertidumbre consta de dos partes: a) propiedades físicas de los materiales, b) propiedades químicas. Este apartado se refiere a como está constituida la formulación del material.

- Incertidumbre de producción, la cual se debe a la operación del proceso teniendo como componentes principales a los operadores y métodos de trabajo, maquinaria, mantenimiento, entre otras.

- Incertidumbre externa generalmente procede de la naturaleza entre los factores más comunes figuran: ambiente, temperatura, iluminación, humedad, radiación.

Los factores ambientales antes mencionados, contribuyen a que el desempeño de los operadores no sea adecuado, puesto que alteran el área de confort donde ellos se desenvuelven y provocan que ese desempeño no sea óptimo, ocasionando que los juicios que emiten y las decisiones que toman no sean acertadas. Los cambios (de diseño de ingeniería, ambientales, entre otros), también provocan incertidumbre en las tres modalidades presentadas en el párrafo anterior [10]. Algunos ejemplos pueden ser encontrados en la literatura acerca del tema de sistemas de procesamiento industrial de imágenes; estos sistemas son usados en diversos campos de la industria y la ciencia que con el paso del tiempo ha evolucionado de la mano de diferentes técnicas a tareas más complicadas pero aún siguen teniendo una serie de restricciones. e.g. identificación de placas, el sistema está restringido a un punto de referencia, se eliminó el número "0" (cero) y la vocal "o" para evitar confusión [14]; clasificación de hojas de tabaco [15] que en este caso la clasificación automática usa solo el $20 \%$ del total de las clases ya que en estas se evalúan varias características, e.g. color, tamaño, forma, superficie y posición (esto es la evaluación de los tallos presentes en las hojas), adicionalmente estas características principales se sub-clasifican e.g. posición se divide en: salientes (X), cortos (C) y hoja sin tallo (B); color: limón (L), naranja $(\mathrm{F})$ y rojo-café $(\mathrm{R})$. Cada grupo de estos es a su vez subdividido en cuatro o cinco sub-grupos debido al tono de la hoja. Esto produce una combinatoria de $\left(3^{\wedge} 2\right)^{\wedge} 5=59049$ posibilidades debido a los subgrupos generados por el tono, el sistema se restringe a evaluar únicamente 24 de las 40 categorías principales debido a que es muy costoso (computacionalmente, matemáticamente y económicamente) para desarrollar un modelo completo.

En las aplicaciones de visión y procesamiento de imágenes existe la restricción de evaluar un pequeño intervalo de las características más comunes debido a que algunas son muy escasas o difíciles de presentarse, por otra parte, si se evaluaran todas las características presentes generarían un crecimiento exponencial en su tamaño, ya que cuando se usan modelos factoriales $n^{k}$ se requiere tener toda la combinatoria del sistema, es decir, tener todos los posibles resultados. Por lo cual se utilizan técnicas 
para reducir el modelo tales como el análisis de componentes principales (PCA). Se puede reducir el tamaño del sistema eliminando las combinaciones que puedan producir confusión tal como en el caso de la identificación de placas con el número 0 y la vocal o. Las limitaciones dentro del modelo de inspección visual provocan que se deba conservar parte del personal altamente entrenado para estudiar los casos que no puedan ser evaluados por el sistema [15]. En este caso se tiene un ejemplo de una muestra que se ubica justo en la frontera de la división entre dos grupos por lo tanto el sistema de clasificación automático reconoce dicha muestra como $50 \%$ perteneciente al grupo 1 y $50 \%$ perteneciente al grupo 2 quedando sin definición particular debido a que no existe esta clase particular dentro del sistema puesto que se eliminó y deberá ser estudiada por personal experto por fuera del sistema de clasificación automática.

El caso de estudio de este artículo se basa en un proceso de inspección para dimensionamiento basado en imágenes y se propone generar un modelo hibrido con diseño factorial y lógica difusa singleton del tipo 1 (T1 SFLS) el cual será validado contra modelos similares de sistemas expertos tales como: Red adaptativa de inferencia neurodifusa (ANFIS) designado en esta caso como T1 ANFIS y Red de función de base radial (RBFN) para verificar su funcionamiento y su precisión. La sección 2 muestra un panorama general de la lógica difusa. En la sección 3 se presenta el modelo del diseño factorial. La sección 4 describe la metodología para crear el modelo hibrido. La sección 5 presenta el caso de aplicación. En la sección 6 se muestran los resultados y finalmente la sección 7 muestra las conclusiones.

\section{$2 \quad$ Lógica Difusa}

La lógica difusa se creó para estudiar las propiedades de los sujetos pertenecientes a un conjunto en el que cada sujeto debe pertenecer a algún conjunto en cierto grado. Para evaluar esta pertenencia [16] presenta un algoritmo matemático para crear aproximaciones por medio de iteraciones vectoriales, en este tipo de evaluación se establece un universo continuo con un número infinito de valores en lugar de uno discreto [17].

Para el diseño de aplicaciones con lógica difusa, se pueden utilizar datos lingüísticos en vez de números o datos crisp que son completamente deterministas [18-20] se basa en reglas condicionadas de la forma (1). Estas reglas son evaluadas por medio de la norma-t o la conorma-t para establecer una evaluación normalizada que se conoce como nivel de activación para cada regla, esta proporcionara una fracción de la evaluación final (2). Posteriormente se ensamblara la función de base difusa (FBF) representada por (3) la cual arrojará un valor difuso de salida que deberá transformarse a un dato crisp o a un valor lingüístico para obtener la aproximación final de la valoración del objeto de estudio,

$$
\begin{gathered}
\text { Regla } n \text { : Si } X_{i} \text { es } A_{i} y \ldots y X_{n} \text { es } A_{n} \text { entonces } Y_{n}=B_{n} \\
i=1,2, \ldots, n
\end{gathered}
$$

donde: $X_{n}$ representa las variables difusas de entrada, $A_{n}$ representa la entrada crisp, $Y_{n}$ representa la salida difusa de la regla y $B_{n}$ representa la salida crisp, 
Sistema inteligente para procesamiento de imágenes en control de calidad basado en el modelo ...

$$
\mu_{R \circ S}(x, z)=\sup _{y \in V}\left[\mu_{R}(x, y) \star \mu_{S}(y, z)\right]
$$

donde: $\star$ denota el producto y $\mu_{R}, \mu_{S}$ representan las funciones de membresía de cada variable, y $\mu_{R \circ S}$ representa el grado de activación de la regla.

$$
F B F=\frac{\sum_{j=1}^{M} \bar{z}^{j}\left(\prod_{i=1}^{n} \mu_{i}^{j}\left(x_{i}\right)\right)}{\sum_{j=1}^{M}\left(\prod_{i=1}^{n} \mu_{i}^{j}\left(x_{i}\right)\right)}
$$

donde: $\bar{z}^{j}$ es la salida de una regla en el espacio $R, \mu_{i}^{j}\left(x_{i}\right)$ representa la activación de la regla.

\section{Diseño factorial}

El diseño factorial [21] es un técnica del DOE, que fue desarrollada para el análisis de factores correlacionados, estos factores presentan limites dentro un universo y son denominados niveles, los cuales se diferencian en alto y bajo (Tabla. 1). Para el establecimiento del modelo general se produce una combinatoria por medio de permutaciones de los factores evaluados en ambos niveles para el caso del modelo $2^{k}$, solo se produce una combinatoria de 4 posibilidades (vértices de la Fig. 1).

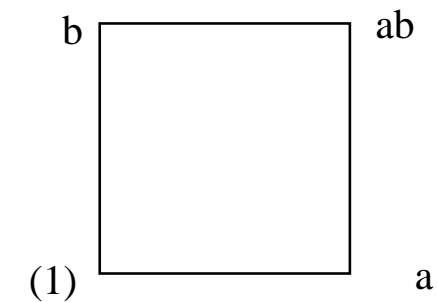

Fig. 1. Representación gráfica del DF $2^{\mathrm{k}}$, adaptado de [21].

Por otra parte, dentro de los límites de la especificación del producto se presentan los máximos y mínimos los cuales se establecerán como los niveles alto y bajo dentro de los tratamientos del DF, estos puntos se interpolan para crear las medias de los conjuntos difusos para las entradas del sistema. Los factores (para el caso del sistema difuso se usan las variables $x_{1}$ y $x_{2}$ ) se denominan $\boldsymbol{A}$ y $\boldsymbol{B}$ en el nivel alto de la especificación, para los niveles bajos de la especificación se omiten. Las combinaciones son descritas con letras, signos, símbolos o números. Los niveles bajos son omitidos con propósitos gráficos. E. g. $\boldsymbol{a} \boldsymbol{b}$ representa las dos variables en nivel alto, $\boldsymbol{b}$ es usada cuando es combinado el nivel alto de $\boldsymbol{B}$ con el nivel bajo de $\boldsymbol{A}, \boldsymbol{a}$ es usada cuando se combinan el nivel alto de $\boldsymbol{A}$ y el nivel bajo de $\boldsymbol{B}, \boldsymbol{I}$ es usado cuando las dos variables se encuentran en el nivel bajo (Fig.1).

La simpleza del modelo de experimento factorial $\left(2^{k}\right)$ se puede expresar matemáticamente como una función lineal multivariada de primer orden (4). Por ejemplo, la función multivariada dada por (5) es llamada el efecto principal debido a que representa las variables de control principales en el proceso, estas son llamadas $\boldsymbol{A}$ 
y $\boldsymbol{B}$. Los efectos principales mencionados se generan por medio de la combinatoria para crear una matriz que representa todas las combinaciones del modelo para el caso del DF/sistema experto solo se producen 4 permutaciones marcando las interacciones de las variables de control del proceso. Las combinaciones de los efectos son llamadas tratamientos y la matriz producida por la combinatoria y sus tratamientos son presentados en la tabla 2. Esta reducción ayuda a que el sistema trabaje en línea sin disminuir su precisión.

$$
\begin{gathered}
a_{1} x_{1} @ a_{2} x_{2} @ \ldots . . . a_{n} x_{n}=y \\
a_{1} x_{1} @ a_{2} x_{2}=y
\end{gathered}
$$

donde: $a_{n}$, es un escalar (coeficiente de la variable independiente) y @ es un operador matemático, e.g. (suma, resta, multiplicación, división, entre otros).

Tabla. 1. Representación simbólica del DF.

\begin{tabular}{|l|l|l|l|}
\hline \multicolumn{2}{|c|}{ Variable } & \multicolumn{2}{c|}{ Efecto } \\
\hline $\boldsymbol{A}$ & $\boldsymbol{B}$ & \multicolumn{2}{|c}{} \\
\hline- & - & Bajo & Bajo \\
\hline+ & - & Alto & Bajo \\
\hline- & + & Bajo & Alto \\
\hline+ & + & Alto & Alto \\
\hline
\end{tabular}

Tabla 2. Entradas del DF, efectos y sus tratamientos.

\begin{tabular}{|l|l|l|l|l|}
\hline \multicolumn{2}{|l|}{ Variable } & \multicolumn{2}{l|}{ Efecto } & Tratamiento \\
\hline $\boldsymbol{A}$ & $\boldsymbol{B}$ & Bajo & Bajo & $\boldsymbol{A}$ Bajo, $\boldsymbol{B}$ Alto \\
\hline $\boldsymbol{A}$ & $\boldsymbol{B}$ & Alto & Bajo & $\boldsymbol{A}$ Alto, $\boldsymbol{B}$ Bajo \\
\hline $\boldsymbol{A}$ & $\boldsymbol{B}$ & Bajo & Alto & $\boldsymbol{A}$ Bajo, $\boldsymbol{B}$ Bajo \\
\hline $\boldsymbol{A}$ & $\boldsymbol{B}$ & Alto & Alto & $\boldsymbol{A}$ Alto, $\boldsymbol{B}$ Alto \\
\hline
\end{tabular}

\section{Metodología}

El proceso metodológico que requiere el sistema de inspección de imágenes necesita de un proceso de adaptación para generar la evaluación en una sola capa debido a que la mayoría las cámaras actuales realizan la adquisición a color se requiere eliminar las capas adicionales. En el caso de matrices RGB (Rojo, verde y azul) 2 capas, 3 capas para matrices YUV (amarillo y ultravioleta) para generar una sola matriz en lugar de un arreglo de matrices, esta se convertirá en una matriz en escala de grises de 8 bits que deberá tratarse para generar una matriz binaria necesaria para la segmentación y la evaluación. El proceso requiere de los siguientes pasos:

1. Adquirir una serie de imágenes de $n$ muestras.

2. Filtrar cada imagen. Por medio de un umbral convertir la imagen a una matriz binaria. 


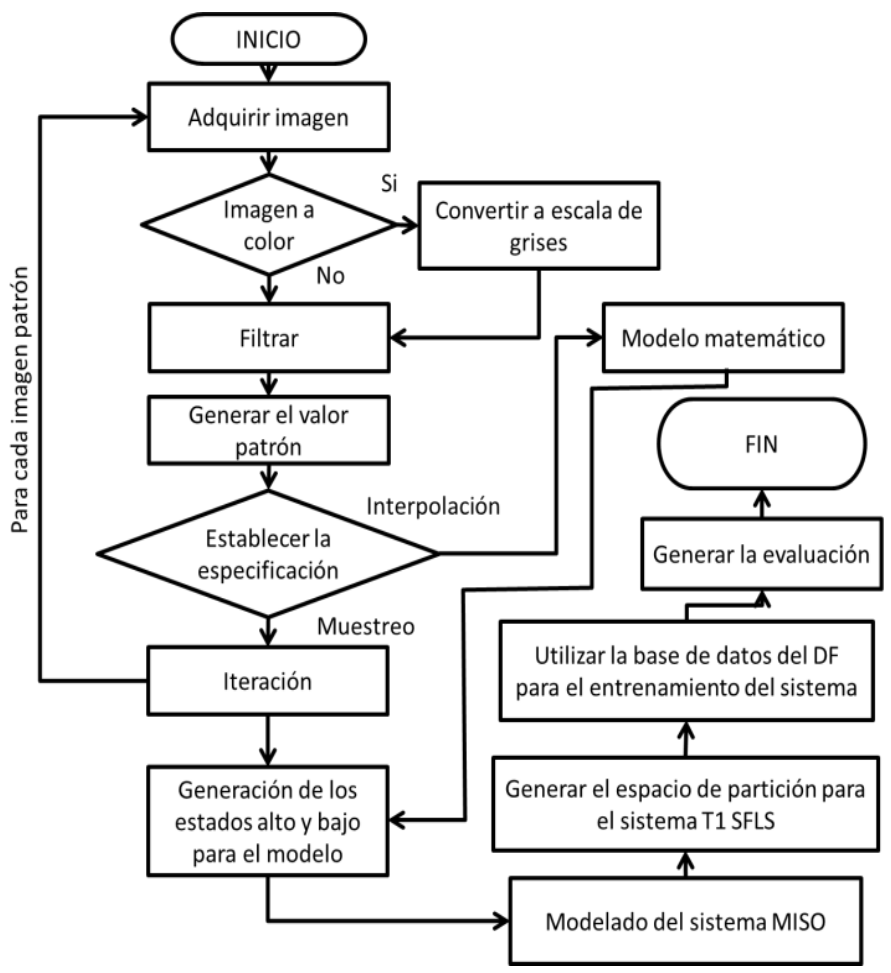

Fig. 2. Diagrama metodológico.

3. Establecer un contador para evaluar la cantidad presente en la muestra en la matriz segmentada.

4. Obtener los límites de la especificación.

5. Por medio de una interpolación del valor obtenido por el contador (generado en el paso 3) y obtener los límites de la especificación en función de lo obtenido en el paso 3.

6. Obtener los límites de la especificación por muestreo de imágenes en los límites de especificación. (repitiendo la metodología varias ocasiones. Una para cada muestra.)

7. Obtener los escalares de los límites para cada variable de los datos obtenidos de los pasos 5 y 6 .

8. Con los valores obtenidos del paso 7 generar un sistema de entradas múltiples y una salida (MISO) que servirá para crear la base de reglas del sistema experto. Estas se obtienen por medio de la ecuación (4) obteniendo los resultados para cada combinación de estados de la tabla 1.

9. Generar un espacio de partición uniforme interpolando los estados alto y bajo de cada variable (Fig. 1), es decir, establecer los vértices del DF como las medias para los conjuntos difusos y su dispersión. 
10. Utilizar la base de datos del DF como datos de entrenamiento para el sistema experto.

11. Generar la evaluación por aproximación.

Con lo puntos anteriores desarrollados se obtiene el sistema experto con la aproximación (T1 SFLS, T1 ANFIS y RFBN) de base de reglas reducida por medio de la metodología denominada sistema experto (SE) SE/DF/ $2^{\mathrm{k}}$. (Fig. 2), cuando la especificación se establece por muestreo, se modelaría el sistema por medio de una base de inferencia individual la cual requiere de múltiples pruebas o iteraciones y posteriormente se hará la reducción de la base de reglas; cuando se establece la especificación por medio de un modelo matemático se deberá generar una interpolación entre la especificación de diseño (sistema CGS, MKS o sistema ingles) para generar unidades en pixeles generando de forma directa un modelo de base de inferencia compuesta con la interpolación de los límites de especificación. La reducción de reglas se da cuando se compara este modelo con un sistema de reglas basado en inferencia individual [5,8]. El sistema de base de inferencia compuesta o base de reglas reducida se genera por medio del modelo SE/DF/2 ${ }^{\mathrm{k}}$. Para realizar la evaluación se repiten los pasos 1 al 3 y 11 para cada muestra a ser evaluada.

\section{Aplicación del sistema experto (T1 SFLS, T1 ANFIS, RBFN)}

La aplicación establecida en el experimento consiste en generar dimensionamiento por medio de imágenes, requiriendo calcular dos parámetros de control dentro de un proceso de producción de placas de base o soporte, la cuales consisten en placas de

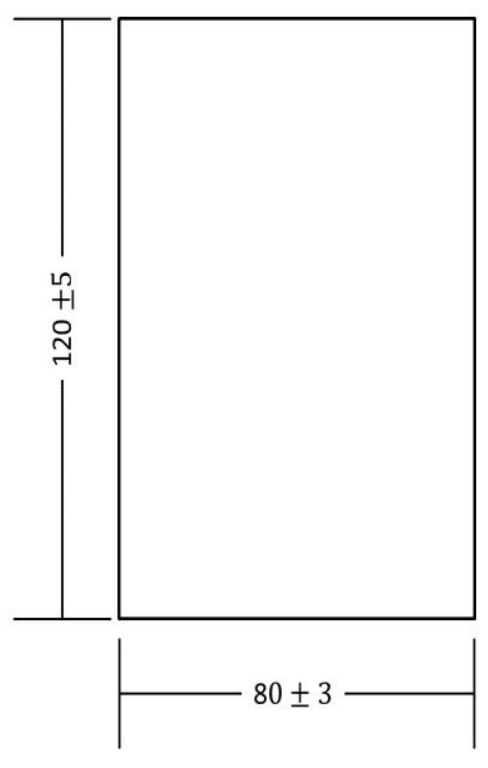

Fig.3. Especificación de la muestra. 
aluminio de 1/16" de espesor con las siguiente especificación (Fig. 3). La imagen obtenida por medio del sistema de visión produce una imagen afectada por la reflexión la cual, ocasiona brillo que provoca que la dimensión se altere, esto se puede observar en la Fig. 4 en la cual aparecen zonas en diferentes tonos de gris en el fondo de la imagen para eliminar esta característica es necesario realizar un preprocesamiento por medio de un filtro el cual funciona por medio de un umbral que realiza el procesado necesario para realizar la conversión a una matriz binaria (Fig. 5). Posteriormente se genera una interpolación de la imagen, y esta se convierte en un vector del cual se obtendrán los valores representativos de cada característica (tabla $3)$. E.g. para generar la interpolación de la matriz numérica que representa la imagen es necesaria (6), la cual representa la característica que dimensiona el ancho de la muestra.

La fase experimental para esta aplicación se ensamblo de la siguiente manera: Todos los experimentos fueron elaborados en una cabina con forma de prisma rectangular de dimensiones $35 \times 35 \mathrm{~cm}$. de base y $55 \mathrm{~cm}$. alto, el equipo de adquisición de imágenes es una cámara web Marca Logitec modelo C110, y el análisis se realizó por medio del software MatLab versión R2009a en una laptop con Windows 7 profesional y un procesador Intel Core i-5 @ $2.5 \mathrm{GHz}$. La iluminación provista al sistema es la siguiente:

Las pruebas del sistema experto (T1 SFLS, T1 ANFIS y RBFN), utilizan un juego de 2 luminarias marca General Electric (GE) colocadas a $10 \mathrm{~cm}$. de la cámara 1 a cada costado, siendo estas focos incandescentes de $75 \mathrm{w}$ con intensidad lumínica de fábrica de 1070 lúmenes por pieza y el objetivo a $43 \mathrm{~cm}$. de la cámara.

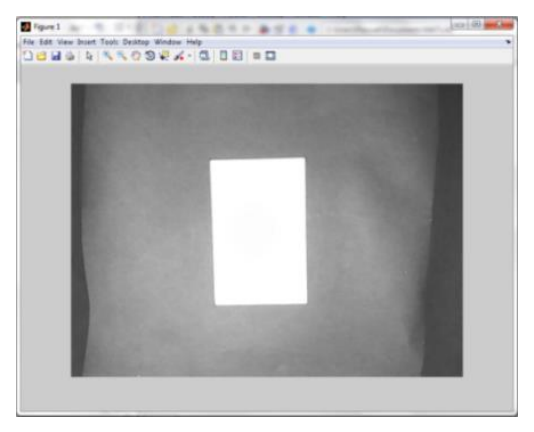

Fig.4. imagen muestra para la inspección.

$$
\sum_{i=1}^{n} \sum_{j=1}^{n} x_{i j} \quad \forall x \in X
$$

A partir de la especificación se obtienen los tratamientos tomando el límite superior de la especificación (muestra 8) y el límite inferior de la especificación (muestra 7 para $x_{1}$, muestra 3 para $x_{2}$ ) para calcular los parámetros $\boldsymbol{A}$ alto y $\boldsymbol{A}$ bajo, así como $\boldsymbol{B}$ alto y $\boldsymbol{B}$ bajo (es necesario ajustar los valores del intervalo de evaluación debido a que generalmente no se pueden derrochar recursos realizando todas combinaciones que marcan las diferentes reglas difusas en pruebas físicas para genera un espacio de partición uniforme), e.g. la diferencia entre la media (muestra 1 para $x_{1}$ y $x_{2}$ ) y el límite superior de especificación (muestra 8 para ambas variables) produce 
un intervalo de 10 unidades para $x_{1}$ y 5 unidades para $x_{2}$, igualmente se deben establecer los valores para los limites inferiores que crearan la matriz de reglas del sistema, esta matriz de reglas basadas en conocimiento funcionan de igual manera para los sistemas: T1 SFLS, T1 ANFIS y RBFN teniendo aquí 3 aproximaciones diferentes de sistemas expertos para los procesos de inspección. Estos límites de la especificación son equivalentes a los tratamientos. Los valores usados para la salida de cada regla difusa son determinados mediante conocimiento experto de evaluación ya sea por un procedimiento o una interpretación de los datos del departamento de calidad. La salida del modelo se generará con el modelo híbrido (SE/DF/2 $\left.2^{\mathrm{k}}\right)$. El DF es usado para crear la base de reglas reducida del sistema experto generando una forma simplificada y teniendo un modelo metodológico específico para la creación de una base de inferencia compuesta por medio del diseño factorial.

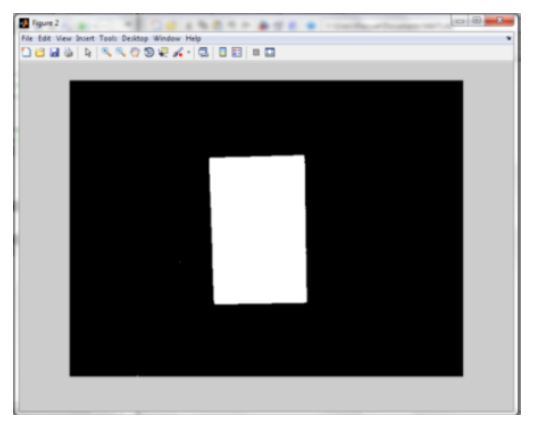

Fig. 5. Imagen filtrada.

La base de datos que aparece en la tabla 3 puede ser usada para modelar el sistema difuso basado en una base de inferencia individual que crearía una base de 13 reglas donde cada prueba o par de datos representaría una regla. Esta se reduce usando los límites mostrados en la Tabla 4. Esta base de inferencia representa al proceso sin la necesidad del conocimiento incierto de una o más personas [22]. El sistema difuso creado utiliza: un modelo tipo Mamdani (T1 SFLS), con un fusificador gaussiano y una implicación de producto, con un defusificador de centro promedio y 4 reglas para el modelo reducido.

Tabla 3. Diseño factorial.

\begin{tabular}{|l|l|l|l|l|l|l|l|l|l|l|l|l|l|}
\hline Muestras & 1 & 2 & 3 & 4 & 5 & 6 & 7 & 8 & 9 & 10 & 11 & 12 & 13 \\
\hline $\mathrm{X}_{1}$ & 208 & 214 & 218 & 218 & 210 & 209 & 205 & 218 & 206 & 211 & 211 & 208 & 207 \\
\hline $\mathrm{X}_{2}$ & 139 & 141 & 135 & 142 & 142 & 141 & 137 & 143 & 141 & 146 & 147 & 143 & 145 \\
\hline Objetivo & 0.6 & 3.6 & 1.2 & 5.4 & 3 & 2.1 & -1.5 & 6 & 1.2 & 5.7 & 6.3 & 3 & 3.9 \\
\hline
\end{tabular}

Tabla 4. Base para la aplicaciones DF/ $2^{k} /$ del sistema expeto (T1 SFLS, T1 ANFIS y RBFN).

\begin{tabular}{|c|c|c|c|c|}
\hline $\mathrm{X}_{1}$ & 133 & 133 & 143 & 143 \\
\hline $\mathrm{X}_{2}$ & 198 & 218 & 198 & 218 \\
\hline $\mathrm{Y}$ & -6 & 0 & 0 & 6 \\
\hline
\end{tabular}




\section{Resultados}

Los diferentes experimentos se basan en comparar el desempeño de diferentes sistemas inteligentes como T1 SFLS, T1 ANFIS y el RBFN muestran que el desempeño del modelo híbrido propuesto es viable para su uso con el beneficio que solo requiere el establecimiento de cuatro reglas derivadas de los puntos axiales del diseño factorial, que a su vez, se obtiene de las especificaciones de fabricación. Las etiquetas para el establecimiento de la partición del universo de propagación de los datos se pueden obtener de los diferentes tratamientos del DF. El modelo hibrido ofrece la ventaja de operar con muy pocas reglas. Se obtienen resultados con una precisión aceptable basada en el diseño factorial. Los resultados de los diferentes experimentos se presentan en la Tabla 5.

Las muestras 3, 4, presentan el caso de una variable cercana al límite de la especificación, debido a esta condición los valores de salida son suavizados o pronunciados, es decir los picos de la función se eliminan o se hacen más pronunciados dependiendo del punto donde estén ubicados en la distribución de los datos, como consecuencia la aproximación generada presentara variaciones contra el objetivo provocando una taza de error del modelo (Fig. 6).

Tabla 5. Muestras evaluadas por medio de la aplicación del sistema experto.

\begin{tabular}{|r|c|c|r|r|r|c|}
\hline Muestra & $\mathbf{X}_{\mathbf{1}}$ & $\mathbf{X}_{\mathbf{2}}$ & Objetivo & T1 SFLS & ANFIS & RBFN \\
\hline $\mathbf{1}$ & 208 & 139 & $\mathbf{0 . 6}$ & 0.2115 & 0.4369 & 0.52707681 \\
\hline $\mathbf{2}$ & 214 & 141 & $\mathbf{3 . 6}$ & 3.3454 & 2.7652 & 1.52429782 \\
\hline $\mathbf{3}$ & 218 & 135 & $\mathbf{1 . 2}$ & 1.4086 & -1.2013 & -1.52429782 \\
\hline $\mathbf{4}$ & 218 & 142 & $\mathbf{5 . 4}$ & 4.78 & 4.1033 & 1.96821401 \\
\hline $\mathbf{5}$ & 210 & 142 & $\mathbf{3}$ & 3.5536 & 2.9742 & 1.96821401 \\
\hline $\mathbf{6}$ & 209 & 141 & $\mathbf{2 . 1}$ & 1.7998 & 2.0809 & 1.52429782 \\
\hline $\mathbf{7}$ & 205 & 137 & $\mathbf{- 1 . 5}$ & -1.0835 & -1.3567 & -0.52707681 \\
\hline $\mathbf{8}$ & 218 & 143 & $\mathbf{6}$ & 4.7123 & 4.8777 & 2.36081604 \\
\hline $\mathbf{9}$ & 206 & 141 & $\mathbf{1 . 2}$ & 0.8261 & 1.6753 & 1.52429782 \\
\hline $\mathbf{1 0}$ & 211 & 146 & $\mathbf{5 . 7}$ & 5.088 & 5.9014 & 3.15864074 \\
\hline $\mathbf{1 1}$ & 211 & 147 & $\mathbf{6 . 3}$ & 6.6451 & 6.5113 & 3.28683149 \\
\hline $\mathbf{1 2}$ & 208 & 143 & $\mathbf{3}$ & 3.8143 & 3.4153 & 2.36081604 \\
\hline $\mathbf{1 3}$ & 207 & 145 & $\mathbf{3 . 9}$ & 4.2509 & 4.6287 & 2.9606785 \\
\hline
\end{tabular}

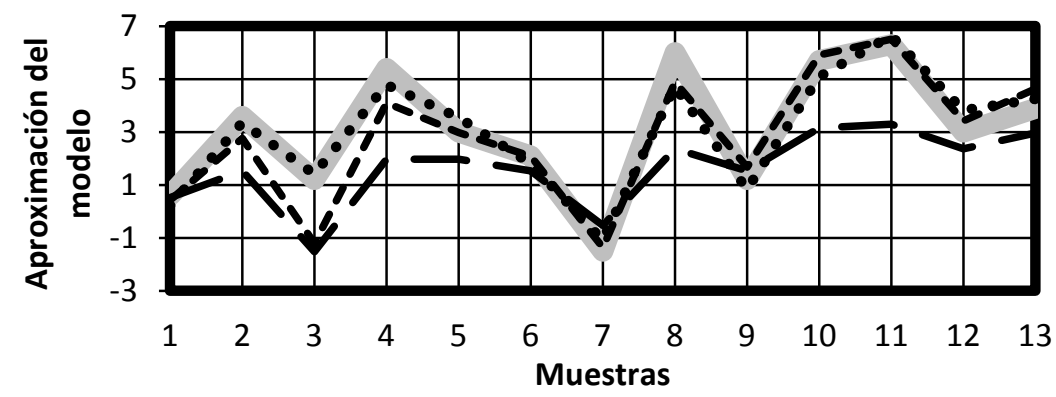

Fig. 6. Resultados de la aproximación generados por el sistema experto. (__ Objetivo, (...) RBFN, (- - ) T1 SFLS, ) T1 ANFIS. 
Las muestras que se encuentran en el intervalo 0.5 a 2.5 desviaciones estándar muestran un desplazamiento proporcional al valor real debido a que la distribución normal en este intervalo presenta un comportamiento lineal, estas variaciones producen pequeños cambios en la salida cuando los cambios en la entrada son significativos. Las muestras encontradas entre la media y 0.5 desviaciones estándar y el intervalo entre 2.5 y 3 desviaciones estándar muestran cambios significativos cuando se producen pequeñas variaciones en la entrada, estas variaciones son producidas debido a la forma de la curva después de los puntos de inflexión, los cuales presentan un comportamiento exponencial. (Fig. 7).

El método desarrollado mejora desempeño de modelos similares tales como: sistema difuso con DCC [4] el cual consta de 11 reglas y base de inferencia individual para la versión de un modelo difuso; por otra parte el modelo (ANFIS/Diseño central compuesto) con base de inferencia compuesta solo opera con 4 reglas obtenidas de los tratamientos del diseño central compuesto [23] y optimiza el modelado del sistema difuso reduciéndolo en más del $60 \%$ debido a que se eliminan 7 de las 11 reglas $(63.63 \%)$.

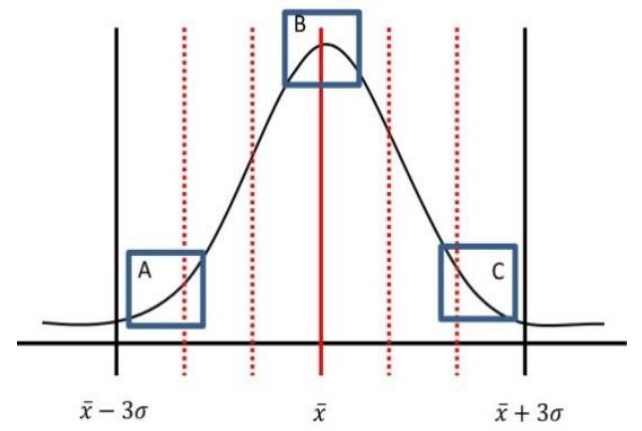

Fig. 7. Puntos de inflexión en una distribución normal (Conjunto difuso).

\section{Conclusiones}

Las propuestas presentadas como modelos híbridos basados en DOE y computación suave operan con una base de reglas obtenidas de múltiples estados basados en la combinatoria de las pruebas y los datos arrojados por el propio DOE.

El modelo propuesto puede reducir la cantidad de reglas, permitiendo que el sistema de inspección trabaje on-line. Debido a que la base de reglas únicamente requiere de 4 reglas para este caso particular el sistema se puede delimitar dentro de un universo específico sin las restricciones de un sistema con limites internos de partición sin las restricciones de un sistema con limites internos de partición dentro del espacio difuso y la aproximación se puede generar en cualquier punto dentro de este universo sin la necesidad de activar múltiples reglas que brindan variaciones por el ajuste y el redondeo que pudiera provocar la función base difusa teniendo la limitante de realizar múltiples cálculos para cada regla y tener que esperar un tiempo de retraso por la aproximación. 
El poder ubicar una muestra en cualquier punto dentro del universo difuso brinda la posibilidad de generar una aproximación de manera rápida solo teniendo que generar el producto por escalar para generar un comportamiento dado en la muestra para cualquier estado del modelo.

Debido a que esta es una investigación en proceso se presentan solo resultados parciales son metodologías basadas en el modelo singleton con sus variantes difusa, neurodifusa y red de base radial puesto que se trabaja en otros modelos capaces de procesar la incertidumbre procedente de las mediciones y esta propuesta como trabajo futuro.

\section{Referencias}

1. Santos, J.F., Rodrigues, L.F.: Applications of a computer visión techniques in the agriculture and food industry: a review. European Food Reserarch and Technology. DOI 10.1007/s00217-012-1844-2 (2012)

2. Czarnowski, I.: Distributed learning with data reduction. Lecture Notes in Computer Science, Transactions on Computational Collective Intelligence IV. 6660, pp. 3-124 (2012)

3. Mendel, J.M. On KM Algorithms for Solving Type-2 Fuzzy Set Problems. IEEE Transactions on Fuzzy Systems, 21 ( 3), pp. 426-446 (2013)

4. Mendel, J.M.: Uncertain Rule-Based Fuzzy Logic Systems: Introduction and New Directions. pp. 130-185, Upper Saddle River, NJ: Prentice-Hall (2001)

5. Wang, L.: A Course in Fuzzy Systems and control, Prentice Hall, 1st. edition (1996)

6. Makadia, A.J., Nanavati, J.I.: Optimisation of machining parameters for turning operations based on response surface methodology. Measurement 46, pp. 1521-1529 (2013)

7. Buragohain, M., Mahanta C.: A novel approach for ANFIS modelling based on full factorial design. Applied Soft Computing, 8, pp. 609-625 (2008)

8. Praga-Alejo, R., González, G. D., Pérez, V.P., Cantú, S.M., Flores, H.B.: Modeling a Fuzzy Logic System Using Central Composite Design. Proceedings of 1st annual world Conference of the Society for Industrial and Systems Engineering. Washington D.C., USA (2012)

9. Martínez, R., Castillo, O., Aguilar, L.T.: Optimization of interval type-2 fuzzy logic controllers for a perturbed autonomous wheeled mobile robot using genetic algorithms. Information Sciences. 179 (13), pp. 2158-2174 (2009)

10. Vale, Z., Morais, H., Faria, P., Ramos, C.: Distribution system operation supported by contextual energy resource management based on intelligent SCADA. Renewable Energy 52, pp. 143-153 (2013)

11. Méndez, G. M., Hernández, M.A.: Hybrid learning mechanism for interval A2-C1type-2 non-singleton type-2 Takagi-Sugeno-Kang fuzzy logic systems. Information Sciences, 220, pp. 149-169 (2013)

12. Tomiyama, T. , Gu, P., Jin, Y., Lutters, D., Kind, Ch., Kimura F.: Design methodologies: Industrial and educational applications. CIRP Annals - Manufacturing Technology, 58. pp. 543-565 (2009)

13. NCS TIB 04-1: National Communications System, Technical Information Bulletin 04-1, Supervisory Control and Data Acquisition (SCADA) Systems, October 2004, Office of the Manager National Communications Systems. Arlington, Virginia. pp. 4-9 (1994) 
14. Ghasemi, M. J., Tajozzakerin, H. R., Omidian, A.R.: An Iranian National Number Plate Localization and Recognition System for Private vehicles. International Journal of Academic Research. 2(6), pp. 13-19 (2010)

15. Zhang, F., Zhang, X.: Classification and Quality Evaluation of Tobacco leaves Based in Image Processing and Fuzzy Comprehensive Evaluation. Sensors. 11(3), pp. 2369-2384 (2011)

16. Zadeh, L.A.: Fuzzy Sets. Information and control, 8, pp.338-353 (1965)

17. Morales-Lunak G.: Introducción a la lógica difusa, Centro de Investigación y Estudios Avanzados del IPN, (CINVESTAV-IPN), [Internet, Consultado el 8 de Octubre de 2011]. (2002)

18. Zadeh, L.A.: The concept of a linguistic variable and its application to approximate reasoning-I, Information Sciences, 8(3), pp. 199-249 (1975)

19. Zadeh, L.A.: The concept of a linguistic variable and its application to approximate reasoning-II, Information Sciences, 8( 4), pp. 301-357 (1975)

20. Zadeh, L.A.: The concept of a linguistic variable and its application to approximate reasoning-III, Information Sciences, 9(1) , pp. 43-80 (1975)

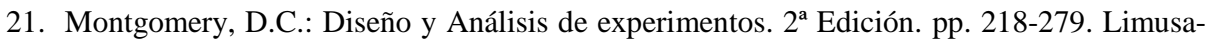
Wiley (2004)

22. Jian-Bo Yang, Jun Liu, Jin Wang, How-Sing Sii, Hong-Wei Wang: Belief Rule-Base Inference Methodology Using the Evidential Reasoning Approach-RIMER, IEEE Transactions on systems, Man and Cybernetics, Part A: Systems and Humans, 36(2), pp. 266-285 (2006)

23. Montes Dorantes, P.N., Praga-Alejo, R., Nieto González, J.P., Méndez, G.M.: Modelado de Sistemas Adaptativos de Inferencia Neuro-Difusa Usando Diseño Central Compuesto. Research in Computing Science, 62, pp. 259-269 (2013) 